\title{
Crossing the wall: branes versus
}

\section{bundles}

\author{
Duiliu-Emanuel Diaconescu and Gregory W. Moore
}

\author{
NHETC and Department of Physics and Astronomy, Rutgers University, \\ Piscataway, NJ 08855-0849, USA \\ duiliu@physics.rutgers.edu, \\ gmoore@physics.rutgers.edu
}

\begin{abstract}
We test a recently proposed wall-crossing formula for the change of the Hilbert space of Bogomol'nyi-Prasad-Sommerfield (BPS) states in $d=4, \mathcal{N}=2$ theories. We study decays of $D 4 D 2 D 0$ systems into pairs of $D 4 D 2 D 0$ systems and we show how the wall-crossing formula reproduces results of Göttsche and Yoshioka on wall-crossing behavior of the moduli of slope-stable holomorphic bundles over holomorphic surfaces. Our comparison shows very clearly that the moduli space of the $D 4 D 2 D 0$ system on a rigid surface in a Calabi-Yau is not the same as the moduli space of torsion-free sheaves, even when worldhseet instantons are neglected. Moreover, we argue that the physical formula should make some new mathematical predictions for a future theory of the moduli of stable objects in the derived category.
\end{abstract}

\section{Contents}

1 Introduction 
3 Wall crossing for Calabi-Yau compactification of type-II strings

4 Moduli spaces 1631

4.1 Generalities 1631

4.2 Wall crossing for moduli spaces of coherent sheaves on a surface $S$

1632

5 Comparison with the physical wall-crossing formula 1637

6 Generalizations

1639

6.1 Bundles on different surfaces

6.2 Bundles on $X: D 6 \longrightarrow D 6+D 6$ 1645

$6.3 \quad D 4 \longrightarrow D 6+\overline{D 6}$ 1645

7 Discussion 1646

Acknowledgments 1647

References

1647

\section{Introduction}

Consider a four-dimensional quantum field theory, or supergravity theory, with $\mathcal{N}=2$ supersymmetry on a spacetime which is asymptotically Minkowskian. These theories have moduli characterizing their vacua as well as distinguished subspaces in their Hilbert space - spaces of Bogomol'nyiPrasad-Sommerfield (BPS) states - defined to be the one-particle states transforming in small representations of the supersymmetry algebra. One of the reasons the spaces of BPS states are so useful and interesting is that the rigidity of the representation theory of supersymmetry implies they are - like an index - immune to many deformations of parameters. Nevertheless, in $d=4, \mathcal{N}=2$ theories closer inspection reveals that the space of BPS states is only locally constant, and in fact it depends on the moduli of the vacuum, a feature which already played an important role in the Seiberg-Witten theory [39]. Spaces of BPS states can jump discontinuously across real codimension one walls in moduli space, known as walls of marginal stability. 
Recently, in the context of Calabi-Yau compactification of type-II string theory, a precise wall-crossing formula for the change in the number of BPS states has been proposed [12]. It is the purpose of this note to test that formula in situations where it is not obvious that the derivation of [12] applies. Conversely, using the relation between BPS states and the mathematics of coherent sheaves and their derived categories, we can use the physically derived formula to make some interesting predictions for mathematics.

Here is a brief outline of the paper: In Section 2, we recall the wallcrossing formula and suggest that it is a universal formula for $d=4 \mathcal{N}=2$ theories. In Section 3, we apply it to the case of wall-crossing for type-II strings on a Calabi-Yau manifold, emphasizing the case where a $D 4$ brane wrapping a surface $S$ splits as a pair of $D 4$ branes wrapping $S$. In Section 4 , we turn to the relation of $D$-branes to mathematical moduli spaces. We review mathematical results on walls of stability for coherent sheaves on surfaces. In Section 5 we compare the physical formula with the results of Göttsche and Yoshioka on wall-crossing formulae for the Hodge polynomials of moduli spaces of coherent sheaves on $S$, in the case where $S$ is rigid. The agreement turns out to be perfect in the leading approximation as the Kähler class goes to infinity. A surprising point emerges that - even neglecting worldsheet instanton corrections - subleading corrections in the expansion in large Kähler class lead to a distinction between the physical and mathematical walls of stability. We interpret this as a signal that the moduli space of $D 4 D 2 D 0$ branes wrapping a rigid surface $S$ is not that of coherent sheaves - as is often asserted - but rather that of stable objects in the derived category. In Section 6, we explore some generalizations which are of interest both physically and mathematically. In particular in Section 6.1 , we discuss decays of $D 4 D 2 D 0$ systems into $D 4 D 2 D 0$ systems wrapping different surfaces. A surprising consequence of these decays is that a $D 4 D 2 D 0$ system can wrap an ample divisor and split into two systems wrapping ample divisors, even at large Kähler structure. ${ }^{1}$ We comment on the implications of this for the Ooguri-Strominger-Vafa (OSV) conjecture in Section 7 , and conclude by pointing out an interesting open problem.

\section{The wall-crossing formula}

Let us recall the basic wall-crossing formula of [12] (whose notation and conventions we always adopt). First, we assume that the BPS state is a particle in a spacetime, which is asymptotically Minkowskian. We assume

\footnotetext{
${ }^{1}$ Examples where an ample $D 4$ decays into a pair of ample $D 4$ 's have been independently discovered in [13].
} 
there is some unbroken abelian gauge symmetry at low energy so that BPS particles can be characterized by their electric and magnetic charge. This charge, which we denote by $\Gamma$, will be valued in a symplectic lattice. The moduli of the vacua will be denoted by $t$, so we are interested in studying the spaces $\mathcal{H}(\Gamma ; t)$ : these are the finite dimensional spaces of BPS one-particle states of charge $\Gamma$ with boundary conditions at infinity corresponding to the vacuum $t$.

The space $\mathcal{H}(\Gamma ; t)$ is a representation of the rotation group $\operatorname{Spin}(3)$. Because of the supersymmetry the representation is of the form

$$
\mathcal{H}(\Gamma ; t)=\left(2(0) \oplus\left(\frac{1}{2}\right)\right) \otimes \mathcal{H}^{\prime}(\Gamma ; t)
$$

In general, we will let $(j)$ denote a representation of $\operatorname{Spin}(3)$ of half-integer spin $j$. We interpret the space for $j=-1 / 2$ as the zero vector space.

Next, we must introduce the Dirac-Schwinger-Zwanziger dualityinvariant symplectic product on the charges, denoted $\left\langle\Gamma_{1}, \Gamma_{2}\right\rangle$. We also need the central charge of the $\mathcal{N}=2$ supersymmetry algebra in the charge sector $\Gamma$ with vacuum determined by $t$. We denote this complex number by $Z(\Gamma ; t)$.

The basic mechanism by which $\mathcal{H}(\Gamma ; t)$ changes was already explained in $[7,8,39]$. There are real codimension one walls of marginal stability, denoted, $M S\left(\Gamma_{1}, \Gamma_{2}\right)$ with $\Gamma=\Gamma_{1}+\Gamma_{2}$ across which those BPS states, which are boundstates of other BPS states of charges $\Gamma_{1,2}$, become unstable. As with non-Fredholm perturbations in index theory, a state can "move off to infinity" in fieldspace and leave the Hilbert space. The walls of marginal stability are therefore defined by

$$
M S\left(\Gamma_{1}, \Gamma_{2}\right)=\left\{t \mid Z\left(\Gamma_{1} ; t\right)=\lambda Z\left(\Gamma_{2} ; t\right) \neq 0, \quad \text { for some } \quad \lambda \in \mathbb{R}_{+}\right\}
$$

Now, a basic stability criterion was derived in $[9,10]$ in the context of $d=$ $4, \mathcal{N}=2$ supergravity: a boundstate which decays across a marginal stability wall will be stable on the side:

$$
\left\langle\Gamma_{1}, \Gamma_{2}\right\rangle \operatorname{Im} Z\left(\Gamma_{1} ; t\right) \overline{Z\left(\Gamma_{2} ; t\right)}>0
$$

The wall-crossing formula then states that as $t$ moves through the wall at $t_{\mathrm{ms}} \in M S\left(\Gamma_{1}, \Gamma_{2}\right)$ from the stable side (2.3) to the unstable side the space 
of BPS states loses a summand

$$
\Delta \mathcal{H}_{\mathrm{BPS}}^{\prime}=\left(j_{12}\right) \otimes \mathcal{H}^{\prime}\left(\Gamma_{1} ; t_{\mathrm{ms}}\right) \otimes \mathcal{H}^{\prime}\left(\Gamma_{2} ; t_{\mathrm{ms}}\right),
$$

where the spin $j_{12}$ is given by

$$
j_{12}=-\frac{1}{2}+\frac{1}{2}\left|\left\langle\Gamma_{1}, \Gamma_{2}\right\rangle\right| .
$$

In stating (2.4), we assume that $\Gamma_{1}$ and $\Gamma_{2}$ are primitive, and that the point $t_{\mathrm{ms}}$ on the wall is generic in the sense that it is not on the intersection of walls of marginal stability for $\Gamma_{i}$ themselves. $^{2}$

Although the formula (2.4) was derived within the specific context of multi-centered solutions of supergravity we believe the wall-crossing formula is, in fact, universal within the context of $d=4, \mathcal{N}=2$ theories and does not depend on being able to represent the boundstate as a classical supergravity solution. On a wall of marginal stability a boundstate of two BPS constituents is marginally bound so the constituents can be adiabatically separated from each other. By locality, the statespace should be a product of the space of states for each constituent times the statespace for the common electromagnetic field. A standard computation in classical electromagnetism shows that two dyons in $\mathbb{R}^{3}$ of charge $\Gamma_{1}, \Gamma_{2}$ carry angular momentum around their midpoint given by

$$
\vec{J}=\frac{1}{2}\left\langle\Gamma_{1}, \Gamma_{2}\right\rangle \frac{\vec{x}_{1}-\vec{x}_{2}}{\left|\vec{x}_{1}-\vec{x}_{2}\right|} .
$$

The "correction" by $-1 / 2$ in (2.5) above is a quantum effect and can be established, in the context of multi-centered solutions of supergravity, as discussed in [11]. It would be desirable to have a more general argument for this quantum correction.

The space of BPS states is not only a representation of $\operatorname{Spin}(3)$ but also of the $U(1) R$-symmetry, where the supercharges have quantum numbers

\footnotetext{
${ }^{2}$ In [12] a generalization for the case when one of $\Gamma_{i}$ is not primitive was proposed. The generalization when both $\Gamma_{1}$ and $\Gamma_{2}$ are not primitive is open and appears to be challenging.
} 


\begin{tabular}{|c||c|c|c|c|}
\hline & $J_{3}$ & $R$ & $J_{3}+R$ & $J_{3}-R$ \\
\hline$Q_{+}$ & $\frac{1}{2}$ & $+\frac{1}{2}$ & 1 & 0 \\
\hline$Q_{-}$ & $-\frac{1}{2}$ & $\frac{1}{2}$ & 0 & -1 \\
\hline $\bar{Q}_{-}$ & $\frac{1}{2}$ & $-\frac{1}{2}$ & 0 & +1 \\
\hline $\bar{Q}_{\dot{+}}$ & $-\frac{1}{2}$ & $-\frac{1}{2}$ & -1 & 0 \\
\hline
\end{tabular}

A useful corollary of (2.4) for our discussion below follows if we define

$$
\Omega(\Gamma ; t ; x, y):=\operatorname{Tr}_{\mathcal{H}^{\prime}(\Gamma ; t)}(-x)^{J_{3}+R}(-y)^{J_{3}-R}
$$

where $J_{3}$ is a generator of $\operatorname{Spin}(3)$ and $R$ is the $U(1) R$-charge of the BPS states.

Now, suppose the modulus $t$ crosses a wall where a particle of charge $\Gamma$ can decay into constituents of charges $\Gamma_{1}$ and $\Gamma_{2}$. The analysis of [11], Section 4.2 shows that all the states can be taken to have zero $R$-charge. ${ }^{3}$ Then the wall-crossing formula (2.4) implies:

$$
\begin{aligned}
\Omega\left(\Gamma ; t_{+} ; x, y\right)-\Omega\left(\Gamma ; t_{-} ; x, y\right)= & (-1)^{\left\langle\Gamma_{1}, \Gamma_{2}\right\rangle-1}(x y)^{-\frac{1}{2}\left(\left\langle\Gamma_{1}, \Gamma_{2}\right\rangle-1\right)} \\
& \times \frac{1-(x y)^{\left\langle\Gamma_{1}, \Gamma_{2}\right\rangle}}{1-x y} \\
& \times \Omega\left(\Gamma_{1} ; t_{\mathrm{ms}} ; x, y\right) \Omega\left(\Gamma_{2} ; t_{\mathrm{ms}} ; x, y\right),
\end{aligned}
$$

where $t_{+}$is on the side $\operatorname{Im}\left(Z_{1} \overline{Z_{2}}\right)>0$ and $t_{-}$is on the side $\operatorname{Im}\left(Z_{1} \overline{Z_{2}}\right)<0$.

Suppose that the states in $\mathcal{H}^{\prime}(\Gamma ; t)$ admit a description as cohomology classes on some moduli space $\mathcal{M}$, which we assume is Kähler and smooth. ${ }^{4}$ A 4D supersymmetric sigma model with Kähler target space $\mathcal{M}$, reduces to a $(2,2)$ supersymmetric quantum mechanics in $0+1$ dimensions. Under the identification of wavefunctions in the quantum mechanics with differential

\footnotetext{
${ }^{3}$ Under the $R$-symmetry $\theta^{\alpha} \longrightarrow \mathrm{e}^{\mathrm{i} \xi} \theta^{\alpha}$ we need to have $W_{\alpha} \longrightarrow \mathrm{e}^{-\mathrm{i} \xi} W_{\alpha}$ and hence $\lambda_{\alpha} \longrightarrow \mathrm{e}^{-\mathrm{i} \xi} \lambda_{\alpha}$. If we take the vacuum $|0\rangle$ in Denef's equation (4.14) to have $R$-charge +1 then all the states in the Coulomb multiplet have $R$-charge 0 .

${ }^{4}$ In the mathematical applications, smoothness is not obvious. In such cases we might be forced to restrict attention to more primitive numerical invariants, such as the Euler character.
} 
forms on $\mathcal{M}$ we have

$$
\begin{aligned}
& Q_{+} \rightarrow \partial, \\
& Q_{-} \rightarrow \bar{\partial}^{\dagger}, \\
& \bar{Q}_{\dot{-}} \rightarrow \bar{\partial}, \\
& \bar{Q}_{\dot{+}} \rightarrow \partial^{\dagger} .
\end{aligned}
$$

In this situation, we can relate $\Omega$ to the Hodge polynomial $e(\mathcal{M} ; x, y)$ of $\mathcal{M}$. We identify $J_{3}$ with the Lefshetz $s l(2)$ acting on the cohomology:

$$
J_{3} \omega=\frac{1}{2}\left(\operatorname{deg} \omega-\operatorname{dim}_{c} \mathcal{M}\right) \omega
$$

and hence

$$
\begin{aligned}
\Omega(\Gamma ; t ; x, y) & =(-1)^{\operatorname{dim} \mathcal{M}}(x y)^{-\frac{1}{2} \operatorname{dim} \mathcal{M}} \sum_{p, q}(-1)^{p+q} x^{p} y^{q} \operatorname{dim} H^{p, q}(\mathcal{M}) \\
& =(-1)^{\operatorname{dim} \mathcal{M}}(x y)^{-\frac{1}{2} \operatorname{dim} \mathcal{M}} e(\mathcal{M} ; x, y) .
\end{aligned}
$$

Evidently, for this equation to make sense, the moduli space $\mathcal{M}$ must depend on $t$.

Two special cases are of particular interest: if we put $x=y$ then we obtain the Poincaré polynomial. If we further take the limit $y \longrightarrow 1$, we obtain the Witten index, i.e., the Euler character of $\mathcal{M}$.

\section{Wall crossing for Calabi-Yau compactification of type-II strings}

Now let $X$ be a compact Calabi-Yau manifold, and consider the compactification of type-IIA strings on $X$. BPS charges are elements of $\gamma \in K^{0}(X)$, but in this paper we will identify the charge with its image in $H^{\text {even }}(X ; \mathbb{Q})$,

$$
\Gamma=\operatorname{ch}(\gamma) \sqrt{\operatorname{Td}(X)}:=r+\operatorname{ch}_{1}(\gamma)+\hat{\operatorname{ch}}_{2}(\gamma)+\hat{\operatorname{ch}}_{3}(\gamma) .
$$

In this case, the symplectic product on charges is given by

$$
\left\langle\Gamma_{1}, \Gamma_{2}\right\rangle=\int_{X} \Gamma_{1} \Gamma_{2}^{*}
$$

where $\Gamma \longrightarrow \Gamma^{*}$ reverses the sign of the components of degree $2 \bmod 4$. The relevant moduli space of vacua for IIA strings is the complexified Kähler 
moduli space, and we identify $t=B+\mathrm{i} J$ where $B$ is the flat $B$-field potential, $B \in H^{2}(X ; \mathbb{R})$ and $J \in H^{2}(X ; \mathbb{R})$ is the Kähler class. In this paper, we will work in the limit of large Kähler class and ignore worldsheet instanton corrections to the period vector. Thus we will identify the holomorphic central charge with

$$
Z_{\mathrm{h}}(\Gamma ; t)=-\int_{X} \mathrm{e}^{-t} \Gamma .
$$

(We only use the central charge to compute walls of marginal stability. Therefore, it suffices to use the holomorphic rather than the normalized central charge. We henceforth drop the subscript $h$.)

In order to compare with mathematical work we will, until Section 6 , concentrate on the case of $D$-branes which are boundstates of $D 4 D 2 D 0$ branes localized on a holomorphic surface $S$ in $X$. As we review in Section 4.1 below these are - classically - the pushforward from $S$ of coherent sheaves $E$ on $S$, or on a "thickening of $S$." We will furthermore take the sheaves on $S$ to be torsion-free.

In this case, the charge is $[28,34]$

$$
\Gamma=\operatorname{ch}\left(j_{*}(E)\right) \sqrt{\operatorname{Td}(X)}
$$

where $j: S \hookrightarrow X$ is the inclusion. Let $c_{1}$ and $c_{2}$ be the Chern classes of $E$ and let $r$ be the rank. It is useful to define

$$
\mu:=\frac{c_{1}}{r}, \quad \Delta:=\frac{1}{r}\left(c_{2}-\frac{r-1}{2 r} c_{1}^{2}\right)
$$

in terms of which

$$
\Gamma=r[S]+r j_{*}(\hat{\mu})+q_{0} \omega,
$$

where $\hat{\mu}=\mu+\frac{1}{2} c_{1}(S)$ and $\omega$ is the unit volume form on $X$. The $D 0$ charge is given by

$$
q_{0}=r\left[\frac{\chi(S)}{24}+\int_{S} \frac{1}{2} \hat{\mu}^{2}-\Delta\right] .
$$

We now consider the wall of marginal stability for a decay $\Gamma \longrightarrow \Gamma_{1}+\Gamma_{2}$ where all three charges $\Gamma, \Gamma_{1}, \Gamma_{2}$ have the form (3.6). For example $\Gamma, \Gamma_{1}, \Gamma_{2}$ could be the charges corresponding to torsion-free sheaves $E, E_{1}, E_{2}$ on $S$, but there are other possibilities, discussed in Section 4.1 below. In this case, the wall of marginal stability can be computed from the vanishing 
locus of 5

$$
\begin{aligned}
\operatorname{Im}\left(Z_{1} \overline{Z_{2}}\right)= & \frac{r_{1} r_{2}}{2} J_{S}^{2} J_{S} \cdot\left(\mu_{1}-\mu_{2}\right)+q_{0}^{1} J_{S} \cdot r_{2} \hat{\mu}_{2}-q_{0}^{2} J_{S} \cdot r_{1} \hat{\mu}_{1} \\
& +\left(J_{S} \cdot B_{S}\right)\left(\frac{r_{1}-r_{2}}{2} J_{S}^{2}+q_{0}^{2}-q_{0}^{1}\right) \\
& +r_{1} r_{2}\left(J_{S} \cdot \hat{\mu}_{1} B_{S} \cdot \hat{\mu}_{2}-J_{S} \cdot \hat{\mu}_{2} B_{S} \cdot \hat{\mu}_{1}\right) \\
& +\frac{r_{1} r_{2}}{2} B_{S}^{2} J_{S} \cdot\left(\mu_{2}-\mu_{1}\right)+\left(J_{S} \cdot B_{S}\right)\left(B_{S} \cdot\left(r_{1} \hat{\mu}_{1}-r_{2} \hat{\mu}_{2}\right)\right) \\
& +\frac{r_{2}-r_{1}}{2} B_{S}^{2}\left(J_{S} \cdot B_{S}\right)
\end{aligned}
$$

where we have organized terms so that each line is homogenous in $B$ and within each line we have written the highest order in $J$ first. In this formula, all the intersection products are computed on $S$. In particular, $J_{S}, B_{S}$ denote the pullbacks of $J, B$ to the surface $S$.

The spin factor is computed from ${ }^{6}$

$$
\left\langle\Gamma_{1}, \Gamma_{2}\right\rangle=r_{1} r_{2} K_{S} \cdot\left(\mu_{2}-\mu_{1}\right)
$$

where $K_{S}$ is the canonical bundle of $S$.

The formulae simplify considerably if we restrict attention to the subspace with $B=0$ and take $J_{S} \longrightarrow \infty$. In this case the marginal stability wall within the Kähler cone of $S$ can be written as

$$
J_{S} \cdot\left(\mu_{1}-\mu_{2}\right)=2\left(\frac{q_{0}^{2}}{r_{2}} \frac{J_{S} \cdot \hat{\mu}_{1}}{J_{S}^{2}}-\frac{q_{0}^{1}}{r_{1}} \frac{J_{S} \cdot \hat{\mu}_{2}}{J_{S}^{2}}\right),
$$

which clearly asymptotes to the wall

$$
J_{S} \cdot\left(\mu_{2}-\mu_{1}\right)=0
$$

for large $J_{S}$.

\footnotetext{
${ }^{5}$ The solution set $\operatorname{Im}\left(Z_{1} \bar{Z}_{2}\right)=0$ consists of both marginal stability and anti-marginal stability walls, the latter being the case where the complex numbers $Z_{1}$ and $Z_{2}$ antialign. The asymptotic component of the wall that we study is a marginal stability wall.

${ }^{6}$ It is important to get the sign right in this formula. Note that for a surface $S \subset X$ and closed differential forms $\eta, \omega$ defined on $S, X$, respectively, we have $\int_{X} \omega \wedge j_{*}(\eta)=$ $\int_{S} j^{*}(\omega) \wedge \eta$. Now, in particular, if $\operatorname{PD}(S)$ is the Poincaré dual of $S$ then we may represent it as the Thom class of the oriented normal bundle. Then, $j^{*}(\operatorname{PD}(S))$ is the Euler class of the normal bundle. For the case of a complex codimension one surface the Euler class is $c_{1}(N(S \subset X))$. Now, because $X$ is Calabi-Yau, $c_{1}(N(S \subset X))=-c_{1}(T S)$. So $j^{*}(\mathrm{PD}(S))=K_{S}$.
} 
The distinction between equations (3.13) and (3.14) is important, and is an order $1 / J$ correction, hence can be significant even when instanton corrections can be neglected. This is a simple way of seeing that it is not sufficient to use the category of coherent sheaves when describing supersymmetric $D$-branes, and presumably the correct generalization is to the derived category of coherent sheaves. We will return to this point in Section 5 .

As a simple example, consider the case of $S=\mathbb{P}^{1} \times \mathbb{P}^{1}$ so that $J_{S}=x d_{1}+$ $y d_{2}$, with $d_{i}$ Poincaré dual to each $\mathbb{P}^{1}$ factor and hence $x, y>0$ in the Kähler cone. We have plotted an interesting example in figure 1.

For large $J_{S}$, Denef's stability conditions says that

$$
K_{S} \cdot\left(\mu_{2}-\mu_{1}\right) J_{S} \cdot\left(\mu_{2}-\mu_{1}\right)>0,
$$

so this is the side on which there are "extra" states which we will lose.

For rigid surfaces, the Poincaré dual $[S]$ will not lie in the Kähler cone, so our discussion here goes beyond that of [12]. (It is possible, but not obvious, that multicentered solutions corresponding to the above splits exist.) We also remark that in equation (3.62) of version 1 of [12], the reader is led to the impression that $D 4$ cannot split into $D 4+D 4$, but the argument there assumes that $[S]$ and $J_{S}$ are proportional to each other.

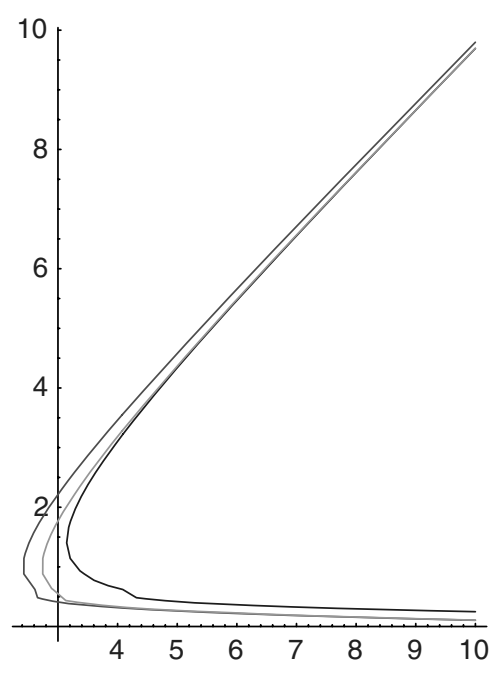

Figure 1: Example of the walls at $B=0$ for different values of $D 0$ charges $q_{0}^{1}, q_{0}^{2}$ with the same total $D 0$ charge. 


\section{Moduli spaces}

\subsection{Generalities}

The classical picture of a supersymmetric type-IIA $D$-brane wrapping cycles in a Calabi-Yau manifold leads one naturally to the identification of classical $D$-brane states with coherent sheaves on $X[29,35]$. However, there is in fact much evidence to suggest that the proper mathematical description of the moduli of classical supersymmetric IIA branes on $X$ is given in terms of the derived category of coherent sheaves [4]. Moduli spaces of derived objects have been constructed so far in certain cases $[32,40]$ using the stability conditions of Bridgeland [6]. Unfortunately, wall-crossing formulae seem to be out of reach at the present stage. Nevertheless, we can make progress for the case of a rigid holomorphic surface $S \subset X$ since then the moduli space of $D 4 D 2 D 0$ branes is expected to be related to the moduli space of slope-stable coherent sheaves. However, as we will see below, even at large $J_{S}$, this is not quite true.

In any case, one should define a moduli space of stable objects of fixed characteristic classes, corresponding to the charge $\Gamma$ and satisfying some $t$-dependent stability condition, $\mathcal{M}(\Gamma ; t)$, and then, ideally, identify

$$
\mathcal{H}^{\prime}(\Gamma ; t) \sim H^{*}(\mathcal{M}(\Gamma ; t)) .
$$

This equation is very rough. In addition to the actual construction of the moduli space, one should specify what kind of cohomology one is using since $\mathcal{M}$ will in general be singular or non-compact, etc.

We can also turn things around, and use the physical formula to make a prediction for wall-crossing behavior of the eventually-to-be constructed moduli spaces of stable objects in the derived category. For example, even if $S$ is not rigid, we expect the moduli space should be fibered over the moduli of holomorphic surfaces $S \subset X$ with fiber given by the moduli of coherent sheaves on $S$ (again, asymptotically for $J \longrightarrow \infty$ ). In this way we make physical predictions for more general moduli spaces of sheaves.

One subtlety we have thus far suppressed is the following. If we consider a charge of the form (3.6) then when $r>1$ the D4-brane charge is not primitive and could in principle be the charge of a "thickening" of the surface $S$. (By a "thickening" we mean that if $S$ is defined locally by the equation $f=0$ then $r S$ is defined by the equation $f^{r}=0$.) Sheaves on such thickenings can, and sometimes do, contribute extra components to the moduli 
space. Physically, this corresponds to solutions to the BPS embedding equations for the $D$-brane gauge field $A$ and normal bundle scalars $\Phi$ in which $\Phi$ is nonzero. Such components have been discussed, for example, in [20]. In our main application in Section 5 below $S$ will be Fano, and using the vanishing theorem of [42] (or an analogous statement in the algebro-geometric version, due to $R$. Thomas) one can show that such components do not occur. Components due to thickenings might be important in the more general applications discussed in Section 6 below. Exploring this point should be interesting, but it is beyond the scope of this paper. ${ }^{7}$

\subsection{Wall crossing for moduli spaces of coherent sheaves on a surface $S$}

Let $S$ be a smooth projective surface with $-K_{S}$ effective, and let $J_{S}$ be in the Kähler cone of $S$. Let $\overline{\mathcal{M}}\left(r, c_{1}, c_{2} ; J_{S}\right)$ be the moduli space of rank $r \geq 1 J_{S}$-semistable torsion-free sheaves on $S$ with Chern classes $\left(c_{1}, c_{2}\right) \in$ $H^{2}(S, \mathbb{Q}) \times H^{4}(X, \mathbb{Q})$. Note that torsion-free sheaves of rank 1 are of the form $\mathcal{I}_{Z} \otimes L$ where $Z$ is a zero-dimensional subscheme of $S$ and $L$ is a line bundle on $S$. Such objects are stable for any polarization $J_{S}$, hence they do not exhibit interesting wall-crossing behavior. ${ }^{8}$ Therefore we will consider $r \geq 2$ from now on. We will denote by $\mathcal{M}\left(r, c_{1}, c_{2} ; J_{S}\right) \subset \overline{\mathcal{M}}\left(r, c_{1}, c_{2} ; J_{S}\right)$ the open subset corresponding to $J_{S}$-stable sheaves.

According to a theorem of Maruyama [33], if $-K_{S}$ is effective, $\mathcal{M}\left(r, c_{1}, c_{2}\right.$; $\left.J_{S}\right)$ is smooth of expected dimension

$$
\operatorname{dim}\left(\mathcal{M}\left(r, c_{1}, c_{2} ; J_{S}\right)\right)=2 r^{2} \Delta-r^{2} \chi\left(\mathcal{O}_{S}\right)+1
$$

where $\Delta$ has been defined in (3.5).

Therefore if any semistable sheaf $E$ with invariants $\left(r, c_{1}, c_{2}\right)$ is automatically stable, it follows that $\overline{\mathcal{M}}\left(r, c_{1}, c_{2}, J_{S}\right)$ is smooth and projective. This will be the case if for example the rank $r$ and the degree $\left(c_{1} \cdot J_{S}\right)$ are coprime (assuming that the polarization $J_{S}$ is integral). In such cases the Hodge polynomial of $\overline{\mathcal{M}}\left(r, c_{1}, c_{2}, J_{S}\right)$ is defined as usual in terms of Dolbeault cohomology.

If there exist strictly semistable objects $E$ with invariants $\left(r, c_{1}, c_{2}\right), \overline{\mathcal{M}}(r$, $\left.c_{1}, c_{2} ; J_{S}\right)$ will be in general singular. However, it turns out that $\overline{\mathcal{M}}\left(r, c_{1}, c_{2}\right.$;

\footnotetext{
${ }^{7}$ We thank Richard Thomas for raising the issue of these thickened components of moduli space.

${ }^{8}$ It is important that we restrict attention to $B=0$ here.
} 
$\left.J_{S}\right)$ is by construction the Geometric Invariant Theory quotient of a closed subscheme $\bar{Q}\left(r, c_{1}, c_{2}\right)$ of the appropriate $Q u o t$ scheme by an algebraic group $G=G L(N)$. According to [43,44], if $-K_{S}$ is effective, the semistable subset $Q\left(r, c_{1}, c_{2}\right) \subset \bar{Q}$ with respect to the linearized group action is smooth. Then one can define a Hodge polynomial of $\overline{\mathcal{M}}\left(r, c_{1}, c_{2} ; J_{S}\right)$ as the equivariant Hodge polynomial of $Q\left(r, c_{1}, c_{2}\right)$ with respect to the $G$-action. More precisely we have

$$
e\left(\mathcal{M}_{H}\left(r, c_{1}, c_{2}\right), x, y\right)=\frac{P_{G}\left(Q\left(r, c_{1}, c_{2}\right), x, y\right)}{1-x y},
$$

where $P_{G}\left(Q\left(r, c_{1}, c_{2}\right), x, y\right)$ denotes the equivariant Hodge polynomial of $Q\left(r, c_{1}, c_{2}\right)$. The normalization factor $1 /(1-x y)$ represents the Hodge polynomial of the classifying space $B \mathbb{C}^{\times}$.

A more powerful approach has been recently developed in $[23,27]$ for moduli spaces equipped with a perfect tangent-obstruction complex. This allows one to define a virtual $\chi_{y}$ genus as well as a virtual elliptic genus. It would be very interesting to understand the physical applications of this construction, but we leave this for future work.

In the following, we will adopt the definition of $[43,44]$ assuming that $-K_{S}$ is effective, and show that the resulting wall-crossing is in agreement with physical predictions.

As shown for example in $[26,41,44]$, the moduli spaces of sheaves depend on the choice of the polarization $J_{S}$ in the Kähler cone and change discontinuously across walls. The dimension does not jump across walls except in cases when the moduli space is empty on one side of a wall. Rather, the two moduli spaces are related by birational transformations.

We will briefly recall some basics of wall-crossing behavior following the treatment of [44], which applies to higher rank sheaves. Employing the notation of [44], let us denote by $\gamma=(r, \mu, \Delta)$, where $\mu, \Delta$ has been defined in (3.5). The marginal stability walls in the Kähler cone $\mathcal{C}(S)$ are in one-toone correspondence to sequences $\gamma_{i}=\left(r_{i}, \mu_{i}, \Delta_{i}\right) \in H^{0}(S, \mathbb{Q}) \oplus H^{2}(S, \mathbb{Q}) \oplus$ $H^{4}(S, \mathbb{Q}), i=1, \ldots, s, s \geq 2$, satisfying the following conditions:

(i) There exists a filtration

$$
0 \subset \mathcal{F}_{1} \subset \cdots \subset \mathcal{F}_{s}=E
$$

with $\gamma\left(\mathcal{F}_{i} / \mathcal{F}_{i-1}\right)=\gamma_{i}, i=1, \ldots, s$.

(ii) There exists $H \in C(X)$ so that $\left(\mu_{i}-\mu_{i-1}, H\right)=0$ for all $i=1, \ldots, s$. (iii) $\Delta_{i} \geq 0$. 
Given such a sequence $\left(\gamma_{1}, \ldots, \gamma_{s}\right)$, the wall $W$ is defined by

$$
W=\left\{H \in C(X) \mid\left(\mu_{i}-\mu_{i-1}, H\right)=0, i=1, \ldots, s\right\} .
$$

A chamber $\mathcal{C}$ is defined to be a connected component of the complement in $\mathcal{C}(S)$ of the union of all walls $W$. Since the moduli space does not vary within any given chamber, we will write $\overline{\mathcal{M}}(\gamma, \mathcal{C})$ for $\overline{\mathcal{M}}\left(r, c_{1}, c_{2}, J_{S}\right)$ with $J_{S} \in \mathcal{C}$.

Suppose $\mathcal{C}_{1}, \mathcal{C}_{2}$ are two chambers in the Kähler cone separated by a wall $W$. Then according to [44], there exist closed subsets $\mathcal{V}_{\mathcal{C}_{a}} \in \overline{\mathcal{M}}\left(\gamma, \mathcal{C}_{a}\right), a=$ 1,2 so that any $\left[E_{1}\right] \in V_{\mathcal{C}_{1}}$ is slope-unstable with respect to any polarization $J_{S, 2} \in \mathcal{C}_{2}$ and conversely any $\left[E_{2}\right] \in V_{\mathcal{C}_{2}}$ is slope-unstable with respect to any polarization in $J_{S, 1} \in \mathcal{C}_{1}$. Moreover, let

$$
0 \subset H N_{1}\left(E_{a}\right) \subset \cdots \subset H N_{h_{a}}\left(E_{a}\right)=E_{a}
$$

be the corresponding Harder-Narasimhan filtrations ${ }^{9}$ of $E_{a} \in V_{\mathcal{C}_{a}}$ for $a=$ 1,2. Then both filtrations have the same length $h_{1}=h_{2}=s$ and the successive quotients satisfy

$$
\gamma\left(\mathrm{HN}_{i}\left(E_{1}\right) / \mathrm{HN}_{i-1}\left(E_{1}\right)\right)=\gamma_{i}, \quad \gamma\left(\mathrm{HN}_{i}\left(E_{2}\right) / \mathrm{HN}_{i-1}\left(E_{2}\right)\right)=\gamma_{s+1-i},
$$

for $i=1, \ldots, s$.

In the following, we will restrict ourselves to walls $W$ corresponding to length $s=2$ filtrations, which is the generic situation. For a wall $W$ separating two chambers $\mathcal{C}_{1}, \mathcal{C}_{2}$, we define $\Gamma_{W}$ to be

$$
\Gamma_{W}=\left\{\begin{array}{l|l}
\left(\gamma_{1}, \gamma_{2}\right) \in\left(H^{\mathrm{ev}}(S, \mathbb{Q})\right)^{2} \mid \begin{array}{l}
\left(\mu_{1}-\mu_{2}, J_{S}\right)=0, \text { for all } J_{S} \in W \\
\left(\mu_{1}-\mu_{2}, J_{S, 2}\right)>0, \text { for all } J_{S, 2} \in \mathcal{C}_{2}
\end{array}
\end{array}\right\},
$$

where $H^{\mathrm{ev}}(S, \mathbb{Q})=H^{0}(S, \mathbb{Q}) \oplus H^{2}(S, \mathbb{Q}) \oplus H^{4}(S, \mathbb{Q})$. Let also

$$
d_{\gamma_{1}, \gamma_{2}}=-r_{1} r_{2}\left(P\left(\mu_{2}-\mu_{1}\right)-\Delta_{1}-\Delta_{2}\right)
$$

\footnotetext{
${ }^{9}$ Any unstable torsion-free sheaf $E$ on a smooth polarized projective variety $X$ admits a canonical filtration $0 \subset \mathrm{HN}_{1}(E) \subset \cdots \subset \mathrm{HN}_{h}(E)=E$ called the Harder-Narasimhan filtration of $E$ [30]. This filtration is inductively constructed so that each successive quotient $\mathrm{HN}_{k}(E) / \mathrm{HN}_{k-1}(E)$ is semistable and moreover $\mathrm{HN}_{k}(E) / \mathrm{HN}_{k-1}(E)$ is the maximal destabilizing subsheaf of $E / \mathrm{HN}_{k-1}(E)$. From a physical point of view, the Harder-Narasimhan filtration encodes the decay products of the unstable $D$-brane configuration described by $E$.
} 
where $P(x)=x \cdot\left(x-K_{S}\right) / 2+\chi\left(\mathcal{O}_{S}\right)$. Then the wall-crossing formula of [44] reads $^{10}$

$$
\begin{aligned}
& e\left(\overline{\mathcal{M}}\left(\gamma, \mathcal{C}_{2}\right), x, y\right)-e\left(\overline{\mathcal{M}}\left(\gamma, \mathcal{C}_{1}\right), x, y\right) \\
&= \frac{1}{1-x y} \sum_{\left(\gamma_{1}, \gamma_{2}\right) \in \Gamma_{W}}\left((x y)^{d_{\gamma_{2}, \gamma_{1}}} e\left(\overline{\mathcal{M}}\left(\gamma_{1}, \mathcal{C}_{2}\right), x, y\right) e\left(\overline{\mathcal{M}}\left(\gamma_{2}, \mathcal{C}_{2}\right), x, y\right)\right. \\
&\left.\quad-(x y)^{d \gamma_{1}, \gamma_{2}} e\left(\overline{\mathcal{M}}\left(\gamma_{1}, \mathcal{C}_{1}\right), x, y\right) e\left(\overline{\mathcal{M}}\left(\gamma_{2}, \mathcal{C}_{1}\right), x, y\right)\right) .
\end{aligned}
$$

Now we specialize to the case of rank $r=2$ sheaves. In this case (4.4) is in agreement with the wall-crossing formula of [26]. In this case, we have

$$
\gamma_{1}=\left(1, F_{1}, n_{1}\right), \quad \gamma_{2}=\left(1, F_{2}, n_{2}\right)
$$

with $n_{1}, n_{2} \in \mathbb{Z}_{\geq 0}$ and $F_{1}, F_{2}$ divisor classes on $S$. In addition $F_{1}+F_{2}=c_{1}$. The corresponding two-term Harder-Narasimhan filtration is

$$
\mathcal{F}_{1}=\mathcal{I}_{Z_{1}}\left(F_{1}\right), \quad E / \mathcal{F}_{1}=\mathcal{I}_{Z_{2}}\left(F_{2}\right),
$$

where $Z_{1}, Z_{2}$ are zero-dimensional subschemes of length $n_{1}, n_{2}$, respectively. The moduli spaces of rank 1 sheaves are insensitive to the chamber structure. We have

$$
\begin{aligned}
& \overline{\mathcal{M}}\left(\gamma_{1}, \mathcal{C}_{1}\right)=\overline{\mathcal{M}}\left(\gamma_{1}, \mathcal{C}_{2}\right)=\operatorname{Pic}(S) \times \operatorname{Hilb}^{n_{1}}(S), \\
& \overline{\mathcal{M}}\left(\gamma_{2}, \mathcal{C}_{1}\right)=\overline{\mathcal{M}}\left(\gamma_{2}, \mathcal{C}_{2}\right)=\operatorname{Pic}(S) \times \operatorname{Hilb}^{n_{2}}(S) .
\end{aligned}
$$

Let $\xi=\mu_{1}-\mu_{2}=2 F_{1}-c_{1}$ as in [26]. Then a straightforward computation yields

$$
\begin{aligned}
& d_{\gamma_{1}, \gamma_{2}}=n_{1}+n_{2}-\frac{1}{2} \xi \cdot\left(\xi+K_{S}\right)-\chi\left(\mathcal{O}_{S}\right), \\
& d_{\gamma_{2}, \gamma_{1}}=n_{1}+n_{2}-\frac{1}{2} \xi \cdot\left(\xi-K_{S}\right)-\chi\left(\mathcal{O}_{S}\right)
\end{aligned}
$$

and one can easily check that the above formula (4.4) is in agreement with Theorem 3.4 of [26].

In this case, we also have a very explicit description of the closed subspaces of the moduli space of rank two sheaves which become unstable when crossing the wall $W^{\xi}=\left\{J_{S} \subset \mathcal{C}(S) \mid \xi \cdot J_{S}=0\right\}$. According to [26] all rank two sheaves which become unstable when crossing the wall $W^{\xi}$ from $\xi \cdot J_{S}<0$ to $\xi \cdot J_{S}>0$ are extensions of the form

$$
0 \rightarrow \mathcal{I}_{Z_{1}}\left(F_{1}\right) \rightarrow \mathcal{E}_{12} \rightarrow \mathcal{I}_{Z_{2}}\left(F_{2}\right) \rightarrow 0 .
$$

\footnotetext{
${ }^{10}$ The wall-crossing formulae of [44] are actually written for Poincaré polynomials. The generalization to Hodge polynomials is straightforward.
} 
For fixed $Z_{1}, Z_{2}, F_{1}, F_{2}$ the isomorphism classes of extensions of the form (4.7) are parameterized by the projective space $\mathbb{P E x t}^{1}\left(\mathcal{I}_{Z_{2}}\left(F_{2}\right), \mathcal{I}_{Z_{1}}\left(F_{1}\right)\right)$ with $^{11}$

$$
\begin{aligned}
K_{12}=\operatorname{dim} \operatorname{Ext}^{1}\left(\mathcal{I}_{Z_{2}}\left(F_{2}\right), \mathcal{I}_{Z_{1}}\left(F_{1}\right)\right) & =-\frac{1}{2} \xi\left(\xi+c_{1}(S)\right)+n_{1}+n_{2}-\chi\left(\mathcal{O}_{S}\right) \\
& =-\frac{1}{2} \xi \cdot c_{1}(S)+c_{2}-\frac{c_{1}^{2}+\xi^{2}}{4}-\chi\left(\mathcal{O}_{S}\right) .
\end{aligned}
$$

The closed subspace of the moduli space which destabilizes when we cross the wall is isomorphic to a closed subvariety $\mathcal{V}_{12}$ of a projective bundle $\mathcal{P}_{12}$

$$
\operatorname{PExt}^{1}\left(\mathcal{I}_{Z_{2}}\left(F_{2}\right), \mathcal{I}_{Z_{1}}\left(F_{1}\right)\right) \longrightarrow \operatorname{Hilb}^{n_{1}}(S) \times \operatorname{Hilb}^{n_{2}}\left(\mathcal{P}_{12}\right) \times \operatorname{Pic}(S) \times \operatorname{Pic}(S) .
$$

Note that not all extensions of the form (4.7) are stable for $\xi \cdot J_{S}<0[26$, Prop. 2.5(3)]. Therefore $\mathcal{V}$ will in general be a proper subvariety of the above projective bundle.

There is a similar closed subspace $\mathcal{V}_{21}$ of the moduli space, which destabilizes when we cross the wall in the opposite direction. This will be isomorphic to a closed subvariety of a projective bundle $\mathcal{P}_{21}$ of the form

$$
\operatorname{PExt}^{1}\left(\mathcal{I}_{Z_{1}}\left(F_{1}\right), \mathcal{I}_{Z_{2}}\left(F_{2}\right)\right) \longrightarrow \mathcal{P}_{21} \operatorname{Hilb}^{n_{1}}(S) \times \operatorname{Hilb}^{n_{2}}(S) \times \operatorname{Pic}(S) \times \operatorname{Pic}(S) .
$$

Although $\mathcal{V}_{12}, \mathcal{V}_{21}$ have in general positive codimension in the projective bundles (4.10), (4.11) respectively, the proof of [26, Thm. 3.4] shows that

$$
\operatorname{dim}\left(\mathcal{V}_{12}\right)-\operatorname{dim}\left(\mathcal{V}_{21}\right)=\operatorname{dim}\left(\mathcal{P}_{12}\right)-\operatorname{dim}\left(\mathcal{P}_{21}\right)
$$

\footnotetext{
${ }^{11}$ The computation here is that $\chi\left(\mathcal{I}_{2}, \mathcal{I}_{1}\right)=\int_{S} \operatorname{ch}\left(\mathcal{I}_{2}^{v}\right) \operatorname{ch}\left(\mathcal{I}_{1}\right) T d(S)$ and $\operatorname{dim} \operatorname{Ext}^{0}=$ $\operatorname{dim} \mathrm{Ext}^{2}=0$.
} 


\section{Comparison with the physical wall-crossing formula}

In order to compare the physical wall-crossing formula with the mathematical results reviewed in the previous section, note that any smooth projective surface with $-K_{S}$ effective can be embedded in a smooth projective CalabiYau three-fold. The most obvious examples are smooth elliptic fibrations with section over $S$. If $-K_{S}$ is effective we can explicitly construct smooth Calabi-Yau Weierstrass models over $S$ which admit a canonical section. Other examples can be obtained by resolving del Pezzo singularities in Calabi-Yau three-folds.

We will compare the physical and mathematical wall-crossing formulae assuming that for each $\gamma_{a}, a=1,2$, the moduli spaces $\overline{\mathcal{M}}\left(\gamma_{a}, \mathcal{C}_{1}\right), \overline{\mathcal{M}}\left(\gamma_{a}, \mathcal{C}_{2}\right)$ are isomorphic to each other. In other words, we will assume that the moduli spaces of the decay products do not change as we cross the wall. This is automatic for decays of rank two sheaves as discussed at the end of the previous section. Then we can denote these moduli spaces simply by $\overline{\mathcal{M}}\left(\gamma_{a}\right), a=1,2$ omitting the polarization subscript. Under this assumption, formula (4.4) becomes

$$
\begin{aligned}
& e\left(\overline{\mathcal{M}}\left(\gamma, \mathcal{C}_{2}\right), x, y\right)-e\left(\overline{\mathcal{M}}\left(\gamma, \mathcal{C}_{1}\right), x, y\right) \\
& \quad=\sum_{\left(\gamma_{1}, \gamma_{2}\right) \in \Gamma_{W}} \frac{(x y)^{d_{\gamma_{2}, \gamma_{1}}-(x y)^{d_{\gamma_{1}, \gamma_{2}}}} e\left(\overline{\mathcal{M}}\left(\gamma_{1}\right), x, y\right) e\left(\overline{\mathcal{M}}\left(\gamma_{2}\right), x, y\right) .}{1-x y} .
\end{aligned}
$$

Using formula (4.2) for the expected dimension of the moduli space, a straightforward computation yields

$$
\begin{aligned}
& \operatorname{dim}\left(\overline{\mathcal{M}}_{H_{a}}(\gamma)\right)-\operatorname{dim}\left(\overline{\mathcal{M}}\left(\gamma_{1}\right)\right)-\operatorname{dim}\left(\overline{\mathcal{M}}\left(\gamma_{2}\right)\right) \\
& \quad=2 d_{\gamma_{2}, \gamma_{1}}+r_{1} r_{2}\left(\mu_{2}-\mu_{1}, K_{S}\right)-1=2 d_{\gamma_{2}, \gamma_{1}}+\left\langle\Gamma_{1}, \Gamma_{2}\right\rangle-1 .
\end{aligned}
$$

Note also that

$$
d_{\gamma_{1}, \gamma_{2}}-d_{\gamma_{2}, \gamma_{1}}=r_{1} r_{2}\left(\mu_{2}-\mu_{1}, K_{S}\right)=\left\langle\Gamma_{1}, \Gamma_{2}\right\rangle .
$$

Then (5.1) can be further rewritten as

$$
\begin{aligned}
e\left(\overline{\mathcal{M}}\left(\gamma, \mathcal{C}_{2}\right), x, y\right)-e\left(\overline{\mathcal{M}}\left(\gamma, \mathcal{C}_{1}\right), x, y\right) & \\
= & \sum_{\left(\gamma_{1}, \gamma_{2}\right) \in \Gamma_{W}}(x y)^{\left(\operatorname{dim}(\overline{\mathcal{M}}(\gamma))-\operatorname{dim}\left(\overline{\mathcal{M}}\left(\gamma_{1}\right)\right)-\operatorname{dim}\left(\overline{\mathcal{M}}\left(\gamma_{2}\right)\right)-\left\langle\Gamma_{1}, \Gamma_{2}\right\rangle+1\right) / 2} \\
& \times \frac{1-(x y)^{\left\langle\Gamma_{1}, \Gamma_{2}\right\rangle}}{1-x y} e\left(\overline{\mathcal{M}}\left(\gamma_{1}\right), x, y\right) e\left(\overline{\mathcal{M}}\left(\gamma_{2}\right), x, y\right)
\end{aligned}
$$


Let us now use the physical formula (2.8) to work out the change of the Hodge polynomial of $\overline{\mathcal{M}}\left(r, c_{1}, c_{2} ; J_{S}\right)$ when $J_{S}$ crosses a wall in the Kähler cone. Of course $\Delta \Omega\left(\Gamma \longrightarrow \Gamma_{1}+\Gamma_{2} ; t_{\mathrm{ms}}\right)$ in general depends on $t_{\mathrm{ms}}$. We are assuming this has a finite limit for $J_{S} \longrightarrow \infty$. From the spin factor we get:

$$
\operatorname{sign}\left(K_{S} \cdot\left(\mu_{1}-\mu_{2}\right)\right)(-1)^{\left\langle\Gamma_{1}, \Gamma_{2}\right\rangle+1}(x y)^{-\frac{1}{2}\left\langle\Gamma_{1}, \Gamma_{2}\right\rangle+\frac{1}{2}} \frac{1-(x y)^{\left\langle\Gamma_{1}, \Gamma_{2}\right\rangle}}{1-x y}
$$

Equation (2.14) yields

$$
e(\overline{\mathcal{M}}(\gamma), x, y)=(-1)^{\operatorname{dim}\left(\overline{\mathcal{M}}\left(\gamma, J_{S}\right)\right)}(x y)^{\frac{1}{2} \operatorname{dim}\left(\overline{\mathcal{M}}\left(\gamma, J_{S}\right)\right)} \Omega(\Gamma ; t, x, y)
$$

for any charge $\Gamma$. Therefore the physical wall-crossing formula (2.8) yields the following prediction for the change in the Hodge polynomials

$$
\begin{aligned}
& \operatorname{sgn}\left(K_{S} \cdot\left(\mu_{1}-\mu_{2}\right)\right)(-1)^{\operatorname{dim}(\overline{\mathcal{M}}(\gamma))-\operatorname{dim}\left(\overline{\mathcal{M}}\left(\gamma_{1}\right)\right)-\operatorname{dim}\left(\overline{\mathcal{M}}\left(\gamma_{2}\right)\right)-\left\langle\Gamma_{1}, \Gamma_{2}\right\rangle+1} \\
& \quad \times(x y)^{\left(\operatorname{dim}(\overline{\mathcal{M}}(\gamma))-\operatorname{dim}\left(\overline{\mathcal{M}}\left(\gamma_{1}\right)\right)-\operatorname{dim}\left(\overline{\mathcal{M}}\left(\gamma_{2}\right)\right)-\left\langle\Gamma_{1}, \Gamma_{2}\right\rangle+1\right) / 2} \\
& \quad \times \frac{1-(x y)^{\left\langle\Gamma_{1}, \Gamma_{2}\right\rangle}}{1-x y} e\left(\overline{\mathcal{M}}\left(\gamma_{1}\right), x, y\right) e\left(\overline{\mathcal{M}}\left(\gamma_{2}\right), x, y\right)
\end{aligned}
$$

The overall sign can be simplified using the second equation in (5.2) which shows that the exponent of $(-1)$ in the first line of $(5.5)$ equals $2 d_{\gamma_{1}, \gamma_{2}}$. Since $d_{\gamma_{1}, \gamma_{2}}$ is an integer by construction (it is actually the codimension of a certain subscheme of a Quot scheme [44]), we are left with

$$
\begin{aligned}
& \operatorname{sgn}\left(K_{S} \cdot\left(\mu_{1}-\mu_{2}\right)\right)(x y)^{\left(\operatorname{dim}(\overline{\mathcal{M}}(\gamma))-\operatorname{dim}\left(\overline{\mathcal{M}}\left(\gamma_{1}\right)\right)-\operatorname{dim}\left(\overline{\mathcal{M}}\left(\gamma_{2}\right)\right)-\left\langle\Gamma_{1}, \Gamma_{2}\right\rangle+1\right) / 2} \\
& \quad \times \frac{1-(x y)^{\left\langle\Gamma_{1}, \Gamma_{2}\right\rangle}}{1-x y} e\left(\overline{\mathcal{M}}\left(\gamma_{1}\right), x, y\right) e\left(\overline{\mathcal{M}}\left(\gamma_{2}\right), x, y\right) .
\end{aligned}
$$

Now let us compare formulae (5.3) and (5.6). In (5.3) there is a sum over all pairs of charges $\left(\gamma_{1}, \gamma_{2}\right)$ in the set $\Gamma_{W}$ associated to a given wall $W$. This reflects the fact that the moduli space undergoes simultaneous birational transformations associated to all possible two term destabilizing HarderNarasimhan filtrations. On the other hand, note that the left-hand side of (3.13) is independent of the choice of distribution $D 0$ brane charge between the two decay products, which is encoded in the invariants $\Delta_{1}, \Delta_{2}$. However, the right-hand side is not independent, and the different torsion free sheaves with the same total second Chern class will in fact lead to different physical stability walls. 
In order to emphasize this point, let us concentrate on moduli spaces of rank 2 sheaves as in [26]. Indeed, in this case, the right-hand side of (3.13) reads:

$$
\frac{1}{J_{S}^{2}}\left(J_{S} \cdot\left(\mu_{2}-\mu_{1}\right)\right)\left(n_{1}+n_{2}\right)+\frac{1}{J_{S}^{2}}\left(J_{S} \cdot\left(c_{1}+c_{1}(S)\right)\right)\left(n_{1}-n_{2}\right)+\cdots,
$$

where $+\cdots$ is independent of $n_{1}$ and $n_{2}$. The dependence of the walls on $n_{1}-n_{2}$ at fixed $n_{1}+n_{2}$ is illustrated in figure 1 .

Thus, already at large radius, taking into account the leading correction in the $1 / J$ expansion, but not including instanton effects, one sees that the relevant physical moduli space cannot be the moduli space of coherent sheaves! We interpret this as a signal that the physical moduli space should be the "moduli space of stable objects in the derived category." As we have mentioned, such a moduli space has not been constructed, and so we can take the physical formula as a prediction for what should be true about such moduli spaces.

Let us finally compare the sign of the wall-crossing formula. Using Denef's stability condition (2.3), which becomes (3.15) in our case, we see that we lose the factorized Hilbert space as we go from

$$
\left(K_{S} \cdot\left(\mu_{1}-\mu_{2}\right)\right)\left(J_{S} \cdot\left(\mu_{1}-\mu_{2}\right)\right)<0 \text { to }\left(K_{S} \cdot\left(\mu_{1}-\mu_{2}\right)\right)\left(J_{S} \cdot\left(\mu_{1}-\mu_{2}\right)\right)>0 .
$$

However, the mathematical wall-crossing formula (5.3) claims a universal result for $\Delta e$ going from

$$
J_{S} \cdot\left(\mu_{1}-\mu_{2}\right)>0 \quad \text { to } \quad J_{S} \cdot\left(\mu_{1}-\mu_{2}\right)<0 .
$$

These are in beautiful agreement, since the spin factor indeed changes sign if we change $K_{S} \cdot\left(\mu_{1}-\mu_{2}\right)<0$ to $K_{S} \cdot\left(\mu_{1}-\mu_{2}\right)>0$. To check the absolute sign note that $K_{S} \cdot\left(\mu_{1}-\mu_{2}\right)<0$ corresponds to going from $J \cdot\left(\mu_{1}-\mu_{2}\right)>$ 0 (this is the moduli space denoted $M_{\mathcal{C}^{\prime}}^{\gamma}$ in Corollary 3.3 of [44]) to $J_{S} \cdot\left(\mu_{1}-\right.$ $\left.\mu_{2}\right)<0$ (this is the moduli space denoted $M_{\mathcal{C}}^{\gamma}$ in Corollary 3.3 of [44]). To compute the $e$-trace over the states we lose we therefore compute $e\left(M_{\mathcal{C}^{\prime}}^{\gamma}\right)-$ $e\left(M_{\mathcal{C}}^{\gamma}\right)$. The agreement between the two formulae is perfect!

\section{Generalizations}

We have argued that the wall-crossing formula is universal, and hence we expect the physical formulae to apply to a wide range of situations which look very different from the mathematical point of view. Here we just point 
out a few special cases where the mathematical counterparts are unknown, but perhaps within reach.

\subsection{Bundles on different surfaces}

We first generalize the story to decays where $\Gamma \longrightarrow \Gamma_{1}+\Gamma_{2}$ involves $D 4$ splitting into a pair of $D 4$ 's, but now the support of the two constituent $D 4$ 's are in different cohomology classes. Thus, we can no longer work within the framework of holomorphic bundles on surfaces, but must consider torsion sheaves within the Calabi-Yau $X$. The surfaces have Poincaré duals denoted by $S_{1}$ and $S_{2}$, respectively. Suppose the surfaces wrap $\Sigma_{1}, \Sigma_{2}$ and let $j_{i}: \Sigma_{i} \longrightarrow X$ be the inclusion.

Now we have

$$
\begin{aligned}
& \operatorname{Im}\left(Z_{1} \overline{Z_{2}}\right)=\frac{r_{1} r_{2}}{2}\left[J_{S_{2}}^{2} J_{S_{1}} \cdot \widehat{\mu}_{1}-J_{S_{1}}^{2} J_{S_{2}} \cdot \widehat{\mu}_{2}\right]+\cdots \\
& \times\left\langle\Gamma_{1}, \Gamma_{2}\right\rangle=r_{1} r_{2}\left(j_{2}^{*}\left(S_{1}\right) \cdot \widehat{\mu}_{2}-j_{1}^{*}\left(S_{2}\right) \cdot \widehat{\mu}_{1}\right) .
\end{aligned}
$$

Even to leading order in $J$ the walls are now in general non-linear and given by

$$
\frac{J_{S_{1}} \cdot \hat{\mu}_{1}}{J_{S_{1}}^{2}}=\frac{J_{S_{2}} \cdot \hat{\mu}_{2}}{J_{S_{2}}^{2}} .
$$

There are many examples in this class because linear systems on compact three-folds generically contain reducible divisors. For concreteness we will consider here decays associated with degenerations of spectral covers in an elliptic fibration $X$. Such divisors are of special interest because they are related to torsion-free sheaves supported on the Calabi-Yau three-fold $X$ by Fourier-Mukai transform. Therefore the physical wall-crossing predictions for spectral covers can be translated to similar statements concerning bundles supported on the Calabi-Yau three-fold $X$.

Let $\pi: X \longrightarrow B$ be a smooth elliptic fibration with a section over a base $B$, where we take $B$ to be a smooth projective surface with effective anticanonical class. We will denote by $\sigma$ the section class on $X$ and write the Kähler form of $X$ in the form

$$
J=t_{f} \alpha_{f}+\pi^{*} J_{B}
$$

where $\alpha_{f}=\sigma+\pi^{*} c_{1}(B)$ is Poincaré dual to the elliptic fiber class. Note that we have the following relations in the intersection ring of $X$

$$
\alpha_{f} \cdot \sigma=0, \quad \alpha_{f}^{2}=\alpha_{f} \cdot \pi^{*} c_{1}(B), \quad \alpha_{f} \cdot \pi^{*} \omega_{1} \cdot \pi^{*} \omega_{2}=\left(\omega_{1} \cdot \omega_{2}\right)_{B}
$$

for any curve classes $\omega_{1}, \omega_{2}$ on $B$. 
Consider the linear system $\left|m \sigma+\pi^{*} \eta\right|$ where $\eta$ is an effective curve class on $B$ and $m \geq 2$. The generic member in this linear system is smooth and irreducible if $\eta$ satisfies the following conditions [16-19,37]:

(i) $|\eta|$ is a base-point free linear system on $B$,

(ii) $\eta-m c_{1}(B)$ is an effective curve class on $B$.

Both these conditions will be satisfied if $\eta$ is a sufficiently ample curve class on $B$. We will assume this to be the case from now on. Note that this also implies that the generic divisor in the above linear system is ample on $X$. We will be interested in moduli spaces of torsion coherent sheaves on $X$ supported on divisors in $\left|m \sigma+\pi^{*} \eta\right|$. These moduli spaces contain closed subsets parameterizing isomorphism classes of sheaves with reducible support. In the following we will show that the sheaves with reducible support can become unstable by crossing certain walls in the Kähler cone of $X$, provided that certain numerical conditions for Chern classes are satisfied.

With this goal in mind let us consider a configuration of two smooth irreducible divisors $\Sigma_{1}, \Sigma_{2}$ with classes $S_{1}=m_{1} \sigma+\pi^{*} \eta_{1}, S_{2}=m_{2} \sigma+\pi^{*} \eta_{2}$ where $m_{1}+m_{2}=m, \eta_{1}+\eta_{2}=\eta$. We will assume that $\Sigma_{1}, \Sigma_{2}$ intersect transversely along a smooth curve in $X$. According to [31], coherent sheaves supported on the union $\Sigma_{1} \cup \Sigma_{2}$ are in one-to-one correspondence with pairs $\left(E_{1}, E_{2}\right)$ of coherent sheaves supported on $\Sigma_{1}, \Sigma_{2}$ respectively and a morphism $f:\left.\left.E_{1}\right|_{\Sigma_{1} \cap \Sigma_{2}} \longrightarrow E_{2}\right|_{\Sigma_{1} \cap \Sigma_{2}}$. Each sheaf $E_{i}$ has topological invariants $\left(r_{i}, \mu_{i}, \Delta_{i}\right)$ as in the previous section for $i=1,2$. We will take $\mu_{1}, \mu_{2}$ to be some generic divisor classes on $S_{1}$, respectively, $S_{2}$ obtained by pull-back from the ambient space

$$
\mu_{1}=b_{1} \sigma+\pi^{*} \rho_{1}, \quad \mu_{2}=b_{2} \sigma+\pi^{*} \rho_{2},
$$

where $b_{1}, b_{2} \in \mathbb{Q}$. Then we have

$$
\begin{aligned}
J_{S_{i}}^{2}= & \left(\eta_{i} \cdot c_{1}(B)\right) t_{f}^{2}+2\left(\eta_{i} \cdot J_{B}\right) t_{f}+m_{i} J_{B}^{2}, \\
J_{S_{i}} \cdot \widehat{\mu}_{i}= & \left(\left(\eta_{i} \cdot \rho_{i}\right)-\frac{1}{2} \eta_{i}^{2}\right) t_{f}+\left(b_{i} \eta_{i}-b_{i} m_{i} c_{1}(B)+m_{i} \rho_{i}\right) \cdot J_{B} \\
& +\frac{1}{2} m_{i}^{2} c_{1}(B) \cdot J_{B}-m_{i} \eta_{i} \cdot J_{B}
\end{aligned}
$$

for $i=1,2$, where all intersection numbers are computed on $B$. Now the walls can be found by substituting equations (6.4) into (6.3). In general we will obtain a fairly complicated cubic equation for the Kähler parameters. 
The spin factor can be computed from

$$
\begin{aligned}
\left\langle\Gamma_{1}, \Gamma_{2}\right\rangle= & r_{1} r_{2} S_{1} \cdot S_{2} \cdot\left[\left(\left(b_{2}-\frac{1}{2} m_{2}\right)-\left(b_{1}-\frac{1}{2} m_{1}\right)\right) \sigma\right. \\
& \left.+\pi^{*}\left(\rho_{2}-\rho_{1}-\frac{1}{2}\left(\eta_{2}-\eta_{1}\right)\right)\right] .
\end{aligned}
$$

In order to simplify the computations let us specialize the discussion to the case $B=\mathbb{P}^{2}$. Let $h$ denote the hyperplane class of $B$. Then we can write

$$
J_{B}=t_{b} h, \quad \eta_{i}=n_{i} h, \quad \rho_{i}=a_{i} h
$$

for some positive integers $n_{i} \in \mathbb{Z}$ and $a_{i} \in \mathbb{Q}, i=1,2$. Equations (6.4) become

$$
\begin{aligned}
J_{S_{i}}^{2} & =m_{i} t_{b}^{2}+3 n_{i} t_{f}^{2}+2 n_{i} t_{b} t_{f} \\
J_{S_{i}} \cdot \widehat{\mu}_{i} & =-\frac{1}{2} n_{i}\left(n_{i}-a_{i}\right) t_{f}+\left(n_{i} b_{i}+\left(a_{i}-3 b_{i}-n_{i}\right) m_{i}+\frac{3}{2} m_{i}^{2}\right) t_{b} .
\end{aligned}
$$

Substituting into (6.3), we obtain the following cubic equation:

$$
\begin{aligned}
& \frac{-\frac{1}{2} n_{1}\left(n_{1}-a_{1}\right) x+\left(n_{1} b_{1}+\left(a_{1}-3 b_{1}-n_{1}\right) m_{1}+\frac{3}{2} m_{1}^{2}\right)}{3 n_{1} x^{2}+2 n_{1} x+m_{1}} \\
& =\frac{-\frac{1}{2} n_{2}\left(n_{2}-a_{2}\right) x+\left(n_{2} b_{2}+\left(a_{2}-3 b_{2}-n_{2}\right) m_{2}+\frac{3}{2} m_{2}^{2}\right)}{3 n_{2} x^{2}+2 n_{2} x+m_{2}},
\end{aligned}
$$

where $x=t_{f} / t_{b}$.

Marginal stability walls will correspond to positive real solutions of equation (6.7). This yields several conditions on the Chern classes which can in principle be satisfied because we have many free parameters $\left(m_{i}, n_{i}, a_{i}, b_{i}\right)$, $i=1,2$. In order to obtain a more tractable equation, let us make a further simplification taking the component $S_{1}$ to be the section of the elliptic fibration, i.e., $m_{1}=1, n_{1}=0$. We will also set $b_{1}=0$. Then $n_{2}=n$, we obtain the quadratic equation

$$
3 n x^{2}+n\left(2+\frac{n-a_{2}}{2 a_{1}+3}\right) x+m_{2}-\frac{2 n b_{2}+2\left(a_{2}-3 b_{2}-n\right) m_{2}+3 m_{2}^{2}}{2 a_{1}+3}=0,
$$

if $a_{1} \neq-3 / 2$, and the linear equation

$$
\frac{1}{2} n\left(n-a_{2}\right) x=\left(n b_{2}+\left(a_{2}-3 b_{2}-n\right) m_{2}+\frac{3}{2} m_{2}^{2}\right),
$$

if $a_{1}=-3 / 2$. 
Now it is clear that these equations will have positive real solutions in a certain range of the parameters $\left(n, m_{2}, a_{1}, a_{2}, b_{2}\right)$. For each such solution the corresponding wall is a straight line in the Kähler cone.

An interesting special case is $m=1$. In this case, all divisors in a linear system of the form $\left|\sigma+\pi^{*} \eta\right|$ are reducible if $\eta \neq 0$. The generic divisor has two components - a horizontal component in class $S_{1}=\sigma$ and a vertical component in class $S_{2}=\pi^{*} \eta$. Let us take

$$
\mu_{2}=b_{2} \sigma+c f,
$$

where $f$ is an elliptic fiber class of the vertical component $\Sigma_{2}$. Note that this is not a generic divisor class obtained by restriction from $X$ as in the previous example. We will keep $b_{1}=0$. Repeating the previous computations we obtain

$$
\begin{aligned}
J_{S_{1}}^{2} & =J_{B}^{2}, \\
J_{S_{2}}^{2} & =\left(\eta \cdot c_{1}(B)\right) t_{f}^{2}+2\left(\eta \cdot J_{B}\right) t_{f}, \\
J_{S_{1}} \cdot \widehat{\mu}_{1} & =\mu_{1} \cdot J_{B}+\frac{1}{2} c_{1}(B) \cdot J_{B}, \\
J_{S_{2}} \cdot \widehat{\mu}_{2} & =-\frac{1}{2} \eta^{2} t_{f}+b_{2}\left(\eta \cdot J_{B}\right)+c t_{f} .
\end{aligned}
$$

Specializing again to the case $B=\mathbb{P}^{2}$ we obtain the quadratic equation

$$
3 n x^{2}+\left(2 n+\frac{n^{2}-2 c}{2 a_{1}+3}\right) x-\frac{2 n b_{2}}{2 a_{1}+3}=0
$$

assuming again $a_{1} \neq-3 / 2$. If $a_{1}=-3 / 2$ are left again with a linear equation

$$
\left(n^{2}-2 c\right) x=2 n b_{2} .
$$

To conclude this section let us briefly translate the above wall crossing predictions into similar statements for torsion-free sheaves on $X$ using the Fourier-Mukai transform $[1,2,5,14,15,24,25]$. According to $[5,25]$ reducible spectral covers correspond to bundles constructed by extensions.

More precisely, suppose the spectral data consist of two smooth irreducible divisors $\Sigma_{1}, \Sigma_{2}$ equipped with spectral line bundles $L_{1}, L_{2}$, and an isomorphism $\left.\left.L_{1}\right|_{\Sigma_{1} \cap \Sigma_{2}} \simeq L_{2}\right|_{\Sigma_{1} \cap \Sigma_{2}}$ as in Section 5 of [5]. The intersection $\left(\Sigma_{1} \cap \Sigma_{2}\right)$ is assumed transverse and smooth. Let $F_{1}, F_{2}$ be the holomorphic bundles on $X$ corresponding to the spectral data $\left(\Sigma_{1}, L_{1}\right),\left(\Sigma_{2}, L_{2}\right)$, respectively. Then the corresponding bundle $F$ on $X$ is obtained by an elementary modification of the form

$$
0 \longrightarrow F \longrightarrow F_{1} \oplus F_{2} \longrightarrow Q \longrightarrow 0,
$$


where $Q$ is a torsion coherent sheaf on $X$ supported on the vertical divisor $\left.D=\pi^{-1}\left(\pi\left(\Sigma_{1} \cap \Sigma_{2}\right)\right)\right)$. $Q$ is essentially the Fourier-Mukai transform of the sheaf $L_{1} \mid \Sigma_{1} \cap \Sigma_{2}$.

The previous computations predict that bundles of the form (6.12) will become unstable as we cross certain walls of marginal stability whenever equation (6.7) admits real positive solutions. In certain cases, such elementary modifications can be equivalently described as extensions. For example suppose that $Q=j_{D *}\left(\left.F_{1}\right|_{D}\right)$ where $j_{D}: D \hookrightarrow X$ is the embedding of $D$ in $X$. Then $F$ is isomorphic to an extension of the form

$$
0 \longrightarrow F_{1}(-D) \longrightarrow F \longrightarrow F_{2} \longrightarrow 0
$$

In these cases, the extensions become unstable when crossing the wall, yielding a higher dimensional analog of the decays studied in the previous sections.

It is very interesting to consider the case $m=1$ from this point of view. In this case, the spectral data consists of a horizontal component $\Sigma_{1}$ identified with the canonical section, and a vertical component $\Sigma_{2}$. We also have line bundles which agree on the intersection as above. Using equations (6.10), (6.8) it is not hard to produce concrete examples of marginal stability walls for such configurations.

As shown in Section 5 of [37], the Fourier-Mukai transform of this spectral data is a rank one torsion free sheaf of the form $\mathcal{I}_{Z} \otimes L$ where $Z$ is a codimension two subscheme of $X$ and $L$ is a line bundle on $X$. Note that the ideal sheaf $\mathcal{I}_{Z} \otimes L$ fits in an exact sequence

$$
0 \longrightarrow \mathcal{I}_{Z} \otimes L \longrightarrow L \longrightarrow \mathcal{O}_{Z} \otimes L \longrightarrow 0
$$

Then our prediction is that $\mathcal{I}_{Z} \otimes L$ will become unstable across the wall, and it will decay into $L$ and $\mathcal{O}_{Z} \otimes L$. This leads to an apparent contradiction since rank one torsion-free sheaves are known to be stable for any values of the Kähler moduli. Here we predict non-trivial wall-crossing behavior even for trivial $B$-field, generalizing the examples found in [12]. Although such decays are impossible in the abelian category of coherent sheaves on $X$, they are very natural from the point of view of $\Pi$-stability in the derived category of $X[3,21,22]$. Indeed, the Fourier-Mukai transform is related to $T$-duality in the physical setup, and hence the Kähler class of the fiber will not be large. Accordingly one cannot neglect worldsheet instanton corrections and one must use $\Pi$-stability. It would be interesting to study this in detail using the rigorous methods developed in [6]. Similar decays of ideal sheaves as well as applications to enumerative geometry are being considered in [38]. 


\subsection{Bundles on $X: D 6 \longrightarrow D 6+D 6$}

Suppose $\mathcal{E} \longrightarrow X$ is a general torsion free sheaf on $X$. We set

$$
\Gamma=\operatorname{ch}(\mathcal{E}) \sqrt{T d(X)}:=r+\operatorname{ch}_{1}(\mathcal{E})+\hat{\operatorname{ch}}_{2}(\mathcal{E})+\hat{\operatorname{ch}}_{3}(\mathcal{E}) .
$$

For decays of a $D 6$ to a pair of $D 6$ branes, the marginal stability wall will be a subset of the vanishing locus of

$$
\begin{aligned}
\operatorname{Im}\left(Z_{1} \overline{Z_{2}}\right)= & \frac{J^{3}}{12}\left(r_{2} J^{2} \operatorname{ch}_{1}\left(\mathcal{E}_{1}\right)-r_{1} J^{2} \operatorname{ch}_{1}\left(\mathcal{E}_{2}\right)\right)+\frac{J^{3}}{6}\left(r_{1} \hat{\mathrm{ch}}_{3}\left(\mathcal{E}_{2}\right)-r_{2} \mathrm{ch}_{3}\left(\mathcal{E}_{1}\right)\right) \\
& +\frac{1}{2} J^{2} \operatorname{ch}_{1}\left(\mathcal{E}_{2}\right) J \hat{\mathrm{ch}}_{2}\left(\mathcal{E}_{1}\right)-\frac{1}{2} J^{2} \mathrm{ch}_{1}\left(\mathcal{E}_{1}\right) J \hat{\mathrm{ch}}_{2}\left(\mathcal{E}_{2}\right) \\
& +J \cdot \hat{\mathrm{ch}}_{2}\left(\mathcal{E}_{2}\right) \hat{\mathrm{ch}}_{3}\left(\mathcal{E}_{1}\right)-J \cdot \hat{\mathrm{ch}}_{2}\left(\mathcal{E}_{1}\right) \hat{\mathrm{ch}}_{3}\left(\mathcal{E}_{2}\right),
\end{aligned}
$$

where we have set $B=0$ for simplicity. The spin is computed from:

$$
\left\langle\Gamma_{1}, \Gamma_{2}\right\rangle=r_{1} r_{2}\left(\mu_{1} \cdot \hat{c h}_{2}\left(\mathcal{E}_{2}\right)-\mu_{2} \hat{\operatorname{ch}}_{2}\left(\mathcal{E}_{1}\right)+\frac{\mathrm{ch}_{3}\left(\mathcal{E}_{1}\right)}{r_{1}}-\frac{\mathrm{ch}_{3}\left(\mathcal{E}_{2}\right)}{r_{2}}\right) .
$$

Suppose now that $r, r_{1}, r_{2}>0$ and suppose that in some region of Kähler moduli space a sheaf $\mathcal{E}_{1}$ of rank $r_{1}$ destabilizes $\mathcal{E}$. That is, we can write

$$
0 \rightarrow \mathcal{E}_{1} \rightarrow \mathcal{E} \rightarrow \mathcal{E}_{2} \rightarrow 0 .
$$

Then the standard slope-stability wall is

$$
\frac{J^{2} \operatorname{ch}_{1}\left(\mathcal{E}_{1}\right)}{r_{1}}=\frac{J^{2} \operatorname{ch}_{1}\left(\mathcal{E}_{2}\right)}{r_{2}}
$$

and the walls given by the vanishing of (6.15) indeed asymptote to this wall, but again, different distributions of $D 0 D 2$ charge between the two constituents lead to distinct walls which all asymptote to a common slopestability wall. Therefore our formula for $\Delta \Omega(x, y)$ gives us some information on the Hodge polynomials of the cohomology classes that are lost and gained across this wall.

\section{$6.3 \quad D 4 \longrightarrow D 6+\overline{D 6}$}

One special case of particular interest, which played an important role in [12], occurs when $r=r_{1}+r_{2}=0$. That is, the decay of a $D 4 D 2 D 0$ system into 
a $D 6 \overline{D 6}$ system. Here, for $B=0$ we have:

$$
\begin{aligned}
\operatorname{Im}\left(Z_{1} \overline{Z_{2}}\right)= & \frac{r_{2} J^{3}}{12} J^{2} \operatorname{ch}_{1}(\mathcal{E})-\frac{r_{2} J^{3}}{6} \hat{\operatorname{ch}}_{3}(\mathcal{E})+\frac{1}{2} J^{2} \operatorname{ch}_{1}\left(\mathcal{E}_{2}\right) J \hat{\operatorname{ch}}_{2}\left(\mathcal{E}_{1}\right) \\
& -\frac{1}{2} J^{2} \operatorname{ch}_{1}\left(\mathcal{E}_{1}\right) J \hat{c h}_{2}\left(\mathcal{E}_{2}\right)+\cdots
\end{aligned}
$$

Since $\operatorname{ch}_{1}(\mathcal{E})$ is an effective class (being Poincaré dual to the cycle where the $D 4$ wraps) the wall cannot extend to infinity, at least not with $B=0$. However, for large $D 0$ charge $\hat{c h}_{3}(\mathcal{E})$ the wall can be brought to the regime of large $J$ where our approximations apply.

\section{Discussion}

In this paper, we have shown that the physical wall-crossing formula applies in a more general context than was used in [12]. In particular, combining it with the description of $D$-branes in terms of coherent sheaves leads to rather non-trivial agreement with wall-crossing formulae in the mathematics literature. Moreover, this discussion suggests some interesting expectations for a future theory of the moduli space of stable objects in the derived category.

One point which should be stressed is the following. The decays discussed in Section 6.1 have a potentially important implication for the OSV conjecture [36], since one can arrange that the $D 4$ branes wrap surfaces $S, S_{1}, S_{2}$ all of which are ample, and yet the decay wall is in the Kähler cone. ${ }^{12}$ This means that

$$
\lim _{J \longrightarrow \infty} \Omega(\Gamma ; B+i J)
$$

is not well-defined, even for $D 4 D 2 D 0$ systems where the $D 4$-brane wraps and ample divisor! The wall-crossing formula shows that the jumps in $\Delta \Omega$ are corrections potentially just as large as the world-sheet instanton corrections in the refined version of the OSV conjecture described in [12]. We defer a careful examination of this possibility to future work.

There is one aspect of our discussion which is quite unsatisfactory. This becomes apparent upon a more detailed examination of which states decay as one crosses the wall. To focus the discussion let us return to the case

\footnotetext{
${ }^{12}$ Note that in the examples studied in Section $5 S$ is not ample. Examples where an ample $D 4$ decays into a pair of ample $D 4$ 's have been independently discovered in [13].
} 
$r=2$ where bundles are destabilized by exact sequences such as (4.7). Let us assume for simplicity that the destabilizing subspaces $\mathcal{V}_{1,2}=\mathcal{P}_{1,2}$ in equations (4.10) and (4.11).

In the mathematical description the change of moduli space is given by a simultaneous blow-down of $\mathbb{C} P^{K_{21}-1}$ and blow-up of $\mathbb{C} P^{K_{12}-1}$. In other words, we lose $K_{12}$ states and gain $K_{21}$ states for a net change of $I_{12}=$ $K_{12}-K_{21}=\left\langle\Gamma_{1}, \Gamma_{2}\right\rangle$ states. In the physical description, on the other hand, a spin $\frac{1}{2}\left(\left|I_{12}\right|-1\right)$ multiplet of BPS states moves off to infinity in fieldspace along a Coulomb branch.

These are very different pictures of what happens to the space of BPS states as $t$ crosses the wall, although both pictures agree on the net change of BPS states. Resolving this puzzle is beyond the scope of the present paper, but we believe the resolution will be important and might have a significant impact upon our understanding of the relation between $D$-branes and constructions in algebraic geometry.

\section{Acknowledgments}

We would like to thank Frederik Denef for collaboration on related matters and for very useful discussions. We would also like to thank Ron Donagi, Michael Douglas, Lothar Göttsche, Juan Maldacena, Tony Pantev and Richard Thomas for discussions. We owe special thanks to Lothar Göttsche and Richard Thomas for making their work available to us prior to publication and to Frederik Denef, Lothar Göttsche and Richard Thomas for comments on the draft. This work was partially supported by the DOE under grant no. DE-FG02-96ER4094 and the NSF under grant no. PHY0555374-2006.

\section{References}

[1] B. Andreas, G. Curio, D. H. Ruiperez and S.-T. Yau, Fourier-Mukai transform and mirror symmetry for D-branes on elliptic Calabi-Yau, [arXiv:math.ag/0012196].

[2] B. Andreas, G. Curio, D. Hernandez Ruiperez and S.-T. Yau, Fibrewise T-duality for D-branes on elliptic Calabi-Yau, J. High Energy Phys. 03 (2001), 020 [arXiv:hep-th/0101129].

[3] P. S. Aspinwall and M. R. Douglas, D-brane stability and monodromy, J. High Energy Phys. 05 (2002), 031 [arXiV:hep-th/0110071]. 
[4] P. S. Aspinwall, T. Bridgeland, A. Craw, M. R. Douglas, M. Gross, A. Kapustin, G. W. Moore, G. Segal, B. Szendrői, and P. M. H. Wilson. Dirichlet branes and mirror symmetry, Clay Mathematics Monographs, 4, American Mathematical Society, Providence, RI, 2009.

[5] M. Bershadsky, A. Johansen, T. Pantev and V. Sadov, On fourdimensional compactifications of F-theory, Nucl. Phys. B505 (1997), 165-201 [arXiV:hep-th/9701165].

[6] T. Bridgeland, Stability conditions on triangulated categories, Ann. Maths. (2), 166(2) (2007), 317-345 [arXiv:math/0212237].

[7] S. Cecotti, P. Fendley, K. A. Intriligator and C. Vafa, A new supersymmetric index, Nucl. Phys. B386 (1992), 405 [arXiv:hep-th/9204102].

[8] S. Cecotti and C. Vafa, On classification of $N=2$ supersymmetric theories, Commun. Math. Phys. 158 (1993), 569 [arXiv:hep-th/9211097].

[9] F. Denef, Supergravity flows and D-brane stability, J. High Energy Phys. 08 (2000), 050 [arXiv:hep-th/0005049].

[10] F. Denef, On the correspondence between D-branes and stationary supergravity solutions of type II Calabi-Yau compactifications, [arXiv:hep-th/0010222].

[11] F. Denef, Quantum quivers and Hall/hole halos, J. High Energy Phys. 10 (2002), 023 [arXiv:hep-th/0206072].

[12] F. Denef and G. W. Moore, Split states, entropy enigmas, holes and halos, [arXiv:hep-th/0702146].

[13] F. Denef and D. Van den Bleeken, Unpublished.

[14] R. Donagi, Heterotic/F-theory duality, [arXiV:hep-th/9802093].

[15] R. Donagi, Taniguchi lecture on principal bundles on elliptic fibrations, [arXiV:hep-th/9802094].

[16] R. Donagi, A. Lukas, B. Ovrut and D. Waldram, Nonperturbative vacua and particle physics in M-theory, J. High Energy Phys. 05 (1999), 018 [arXiV:hep-th/9811168].

[17] R. Donagi, A. Lukas, B. Ovrut and D. Waldram, Holomorphic vector bundles and nonperturbative vacua in M-theory, J. High Energy Phys. 06 (1999), 034 [arXiv:hep-th/9901009].

[18] R. Donagi, B. Ovrut, T. Pantev and D. Waldram, Standard models from heterotic M-theory, Adv. Theor. Math. Phys. 5 (2002), 93 [arXiv: hep-th/9912208].

[19] R. Donagi, B. Ovrut, T. Pantev and D. Waldram, Standard model bundles, Adv. Theor. Math. Phys. 5 (2002), 563 [arXiv:math. AG/0008010].

[20] R. Donagi, S. Katz and E. Sharpe, Spectra of D-branes with Higgs vevs, Adv. Theor. Math. Phys. 8 (2005), 813 [arXiv:hep-th/0309270]. 
[21] M. R. Douglas, D-branes, categories and $N=1$ supersymmetry, J. Math. Phys. 42 (2001), 2818-2843 [arXiV:hep-th/0011017].

[22] M. R. Douglas, B. Fiol and C. Romelsberger, Stability and BPS branes, J. High Energy Phys. 09 (2005), 006 [arXiV:hep-th/0002037].

[23] B. Fantechi and L. Göttsche, Riemann-Roch theorems and elliptic genus for virtually smooth schemes, [arXiv:0706.0988].

[24] R. Friedman, J. Morgan and E. Witten, Vector bundles and F theory, Commun. Math. Phys. 187 (1997), 679-743 [arXiV:hep-th/9701162].

[25] R. Friedman, J. W. Morgan and E. Witten, Vector bundles over elliptic fibrations, [arXiV:alg-geom/9709029].

[26] L. Göttsche, Change of polarization and Hodge numbers of moduli spaces of torsion free sheaves on surfaces, Math. Z. 223 (1996), 247.

[27] L. Göttsche, H. Nakajima and K. Yoshioka, Donaldson= Seiberg-Witten from Mochizuki's formula and instanton counting, Publ. Res. Inst. Math. Sci. 47(1) (2011), 307-359.

[28] M. B. Green, J. A. Harvey and G. W. Moore, I-brane inflow and anomalous couplings on D-branes, Class. Quant. Grav. 14 (1997), 47 [arXiv:hep-th/9605033].

[29] J. A. Harvey and G. W. Moore, On the algebras of BPS states, Commun. Math. Phys. 197 (1998), 489 [arXiv:hep-th/9609017].

[30] D. Huybrechts and M. Lehn, The geometry of moduli spaces of sheaves, Aspects of Mathematics, E31. Friedr. Vieweg \& Sohn, Braunschweig, 1997.

[31] M.-A. Inaba, On the moduli of stable sheaves on a reducible projective scheme and examples on a reducible quadric surface, Nagoya Math. J. 166 (2002), 135.

[32] M. Inaba, Moduli of stable objects in a triangulated category, [arXiv:math/0612078].

[33] M. Maruyama, Moduli of stable sheaves. II, J. Math. Kyoto Univ. 18 (1977), 557.

[34] R. Minasian and G. W. Moore, K-theory and Ramond-Ramond charge, J. High Energy Phys. 11 (1997), 002 [arXiv:hep-th/9710230].

[35] D. R. Morrison, The geometry underlying mirror symmetry, [arXiv:alg-geom/9608006].

[36] H. Ooguri, A. Strominger and C. Vafa, Black hole attractors and the topological string, Phys. Rev. D70 (2004), 106007 [arXiv: hep-th/0405146].

[37] B. Ovrut, T. Pantev and J. Park, Small instanton transitions in heterotic M-theory, J. High Energy Phys. 05 (2000), 045 [arXiv:hep-th/0001133]. 
[38] R. Pandharipande and R. P. Thomas, Curve counting via stable pairs in the derived category. Invent. Math. 178(2) (2009), 407-447.

[39] N. Seiberg and E. Witten, Electric-magnetic duality, monopole condensation, and confinement in $N=2$ supersymmetric Yang-Mills theory, Nucl. Phys. B426 (1994), 19 [Erratum — Nucl. Phys. B430 (1994), 485] [arXiv:hep-th/9407087].

[40] Y. Toda, Moduli stacks and invariants of semistable objects on K3 surfaces, [arXiv:math/0703590].

[41] Z. Qin, Chamber structures of algebraic surfaces with Kodaira dimension zero and moduli spaces of stable rank two bundles, Math. Z. 207(1) (1991), 121.

[42] C. Vafa and E. Witten, A strong coupling test of $S$ duality, Nucl. Phys. B431(3) (1994), 3-77 [arXiv:hep-th/9408074].

[43] K. Yoshioka, The Betti numbers of the moduli space of stable sheaves of rank 2 on a ruled surface, Math. Ann. 302(3) (1995), 519.

[44] K. Yoshioka, Chamber structure of polarizations and the moduli of stable sheaves on a ruled surface, Int. J. Math. 7(3) (1996), 411 [arXiv:alg-geom/9409008]. 